\title{
Action co-representation and the sense of agency during a joint Simon task: comparing human and machine co-agents
}

\author{
Aïsha Sahaii $^{1,2}$, Andrea Desantis ${ }^{2,3}$, Ouriel Grynszpan ${ }^{4}$, Elisabeth Pacherie ${ }^{1}$, Bruno \\ Berberian $^{2}$ \\ ${ }^{1}$ Institut Jean-Nicod, Département d'Etudes Cognitives, ENS, EHESS, CNRS, PSL Research \\ University, Paris, France \\ ${ }^{2}$ ONERA, The French Aerospace Lab, Département Traitement de l’Information et Systèmes, \\ Salon-de-Provence, France \\ ${ }^{3}$ Laboratoire Psychologie de la Perception, CNRS UMR 8242 \& Université Paris Descartes, \\ Paris, France \\ ${ }^{4}$ Institut des Systèmes Intelligents et de Robotique, Université Pierre et Marie Curie, Paris, \\ France \\ * Correspondence: \\ PhD student Aïsha Sahaï \\ aisha.sahai@onera.fr
}

\begin{abstract}
Recent studies have suggested that individuals are not able to develop a sense of joint agency during joint actions with automata. We sought to examine whether this lack of joint agency is linked to individuals' inability to co-represent the automaton-generated actions. Fifteen participants observed or performed a Simon response time task either individually, or jointly with another human or a computer. Participants reported the time interval between their response (or the co-actor response) and a subsequent auditory stimulus, which served as an implicit measure of participants' sense of agency. Participants' reaction times showed a classical Simon effect when they were partnered with another human, but not when they collaborated with a computer. Furthermore, participants showed a vicarious sense of agency when co-acting with another human agent but not with a computer. This absence of vicarious sense of agency during human-computer interactions and the relation with action corepresentation are discussed.
\end{abstract}


Key words: joint Simon task, action co-representation, sense of agency, cooperation, humancomputer interaction

\section{Introduction}

The sense of agency refers to the experience of controlling one's own actions, and through them, events in the outside world (Haggard \& Chambon, 2012). This form of selfawareness is the basis of the legal and ethical structure of modern societies and forms the basis on which humans experience responsibility (Moretto, Walsh, \& Haggard, 2011; Frith, 2014; Caspar, Christensen, Cleeremans, \& Haggard, 2016). Interestingly, studies have shown the emergence of a form a shared control when engaged in a cooperative task: we develop an experience of agency for actions and outcomes generated by our partner, a phenomenon called we-agency. This experience of agency could support our engagement in the cooperative task. Given that engagement with automated systems is essential in the context of human-machine cooperative activities such as piloting an airplane, understanding the challenges involved in developing a sense of shared agency with automata becomes a critical concern. The present study aims at addressing this issue and in particular at investigating the factors contributing to our experiences of vicarious agency during joint tasks with humans and artificial agents.

\section{The sense of agency in joint actions}

During the last decades, the sense of agency has largely been investigated using both explicit and implicit measures (for a recent review see Haggard, 2017). For instance, one of the implicit measures most widely used to quantify people's sense of agency is the Intentional Binding phenomenon (IB; see Moore \& Obhi, 2012): when a voluntary movement generates a sensory outcome, people experience an illusory temporal compression between the movement and the outcome. Importantly, this temporal binding seems to reliably occur in situations in which the participant is the agent, but not with passive movements (Haggard, Clark, \& Kalogeras, 2002).

A large body of studies have used IB to shed light on how the sense of agency emerges during joint actions (Capozzi, Becchio, Garbarini, Savazzi, \& Pia, 2016; Obhi \& Hall, 2011a; Strother, House, \& Obhi, 2010). On the one hand, it has been shown that people can exhibit IB for actions and outcomes generated by another human agent when they are engaged in a cooperative task (Obhi \& Hall, 2011a; Strother, House, \& Obhi, 2010). For instance, in 
Strother and colleagues' (2010) study, pairs of participants were seated in front of a monitor and each member of the pair had his/her right finger on one side of the spacebar. The fingers of the participants were hidden so that no visual information about who was pressing the key was available. Participants alternately pressed the spacebar. During the operant trials, the key press triggered a tone $200 \mathrm{~ms}$ later. Participants had to estimate the perceived moment of the key press or the tone occurrence. By computing the action estimate and the tone estimate into one interval, the authors observed intentional binding for the other-generated shared action in a nearly similar fashion as for the self-generated action (Strother, et al., 2010). In addition, in Obhi \& Hall’s (2011a) study, participants were divided in pairs and were asked to press a spacebar. In a first experiment, the two participants could initiate the press at the time of their own choosing, but whenever one participant pressed the spacebar first, the other was to join in and press the spacebar as soon as possible. In a second experiment, the roles of the participants were pre-defined: one of the participants was to press the spacebar first while the second was always following her/his move. In both experiments, participants had to report their feeling of causal responsibility (i.e., their experience of agency) using a percentage scale and also to judge the onset time of the first key press or the onset of the tone. Interestingly, in both experiments, although only the initiator reported a reliable feeling of causal responsibility, both individuals showed IB (Obhi \& Hall, 2011a). Notably, participants experienced IB for their own actions but also for the actions executed by the partner, suggesting that during joint tasks individuals can develop a vicarious sense of agency. It must be emphasized that it is the mere belief that another human agent caused an outcome (and not the fact of seeing or not the human-generated action) that allows individuals to develop a vicarious sense of agency.

On the other hand, there are studies that showed that intentional binding was not socially shared during cooperative tasks (Capozzi, Becchio, Garbarini, Savazzi, \& Pia, 2016; Pfister, Obhi, Rieger, \& Wenke, 2014). For example, Capozzi and colleagues (2016) asked participants to perform a key press that triggered after $259 \mathrm{~ms}$ (T1) an auditory tone that served as a start signal for a (non-naïve) co-agent key press. This co-agent key press triggered a different auditory tone which occurred at a fixed delay of $629 \mathrm{~ms}$ after T1 (T2). In the cooperative condition, they were told to coordinate their actions as if they wanted to create a melody with the two tones. In the competitive condition, the participants were told that the coagent had to perform his key press as fast as possible as if he wanted to "wipe out" T1. Participants had to verbally report their perceived duration of either T1 or T2. The authors found that during sequential joint actions with a co-agent, individuals demonstrated IB for 
their self-generated actions but not for their partner's action, independently of the context of the performed action (Capozzi, et al., 2016). In addition, in Pfister et al. (2014)'s study, participants were paired. One assumed a leader role and the other a follower role. The leader had to press a key at the moment of their own choice and the key press triggered a tone after a certain delay (interval 1). This tone served as a go-signal for the follower to press his or her own key (interval 2). The follower's key press could trigger a tone after a random delay (interval 3) or no tone at all. Both the leader and the follower had to verbally judge the interval lengths. The results showed that the leader's interval estimations were always shorter than the follower's interval estimations, meaning that the leaders always made more IB than the followers. In addition, as the initiator of the action, the leader made IB while the follower, as the observer, did not (interval 1). For the interval 2 estimation, the leader experienced IB for the follower's action but the follower did not. Hence, leaders' sense of agency does not only concern their own actions and their adjacent effect but also predictable actions of other agents. Finally, when the follower's key press generated a sensory consequence, the temporal interval estimations (interval 3) were similar for the leader and the follower: none of them exhibited IB. That is, the follower never experienced a sense of agency whether it be over their own action and its effect or over those of the leader (Pfister, et al., 2014).

Interestingly, with regard to automata, there are studies that have consistently found that individuals' sense of self-agency and vicarious agency were degraded when interacting with a non-human-like artificial agent (e.g. a computer). For example, Obhi and Hall (2011b) showed that in a joint task participants exhibited IB for their own actions and those generated by another human co-agent, whereas, when acting jointly with a machine, IB for their own actions and those generated by the machine disappeared. In a similar vein, Berberian and colleagues (Berberian, Sarrazin, Le Blaye \& Haggard, 2012) showed that human operators experienced a very strong decrease of their sense of agency when interacting with highly automated autopilot interfaces. Moreover, they reported a lack of control over the outcomes generated by the automated system. Conversely, it has been shown that observing an action performed by a human-like automaton (e.g. an anthropomorphic hand with servo-actuated tendons rotating each finger joint) induced a temporal binding effect in a similar way as during the observation of another person performing the same action (Khalighinejad, Bahrami, Caspar \& Haggard, 2016).

Taken together these studies suggest that the ability to build a sense of vicarious agency during a joint task with another agent is highly influenced by the nature of the agent one interacts with. During joint tasks with non-human-like automated machines people fail 
not only to experience the agency of the artificial co-actor but also their own agency. This decrease of agency during human-machine interactions might lead in turn to a reduction of involvement with the automaton since a felt lack of control would directly impact the operator's engagement and responsibility in the task (Moretto, Walsh, \& Haggard, 2011; Frith, 2014; Caspar, Christensen, Cleeremans, \& Haggard, 2016).

This study aims at exploring this decrease in individuals' sense of agency during their interactions with automated agents compared to human-human interactions. Past research suggested that the experience of agency for the actions performed by another individual is based on the ability to simulate or co-represent that individual's motor plans and intentions (Obhi \& Hall, 2011b; Wohlschläger, Engbert, \& Haggard, 2003; see also Kunde, Weller, \& Pfister, 2017). Based on this hypothesis, people would not experience a sense of vicarious agency when interacting with computers because they fail to simulate computer-generated actions (Obhi \& Hall, 2011b). In line with this assumption, the present study investigated the link between action co-representation and individuals' experience of agency during actions performed by another human versus an automated artificial agent in a joint task. The ability to co-represent the actions of the other agent was investigated with the Social Simon task (Sebanz, Knoblich, \& Prinz, 2003)

\section{Social Simon effect: action co-representation in joint actions}

Action co-representation of human-generated actions has been classically demonstrated by the Social Simon Effect (SSE; Sebanz, et al., 2003). In the classical Simon effect people's reaction time in response to the presentation of a target stimulus decreases when the target is presented in the same relative spatial location as the response. For instance, in one version of this paradigm participants are required to execute a left hand action as soon as a red target is presented and a right hand action as soon as a green target it presented. Red/green targets are displayed in either the same relative location as the participant's response (e.g., the target is presented to the left of a central fixation and participants have to perform a left key-press) or in the opposite location (e.g., participants have to press the right key but the target is presented on the left). It has been shown that people are faster and more accurate when the target is on the same side as the response compared to when it is presented on the opposite side. Sebanz et al. (2003) showed with a go no-go task, that when participants had to respond to only one target (e.g., respond only to the green target with the right hand) the interference disappeared. This go no-go task suggested that the Stimulus-Response congruency effect observed in the classical Simon task derives from the cognitive interference 
caused by two different action representations that are concurrently activated (Simon \& Wolf, 1963). However, interestingly, when the participant performs the same go no-go task with another agent (joint Simon task), and each of them is required to respond to only one target (i.e., the participant responds only to the green target, and the partner only to the red target), the interference effect for the incongruent Stimulus-Response key mapping reappeared. Hence, it has been proposed that during a joint task, individuals integrate the behavior of their partner into their own motor scheme even though it is not task relevant (Sebanz, et al., 2003), but see the reference coding hypothesis (Dolk, et al., 2011; Dolk, Hommel, Prinz, \& Liepelt, 2013) or the task representation hypothesis (Yamaguchi, Wall, \& Hommel, 2018). Consistently, neuroimaging data showed that when someone observes or imagines a conspecific performing an action, their premotor cortex is activated - though to a lesser extent than during action execution - allowing action understanding and prediction with the help of the observer's own motor expertise (Calvo-Merino, Glaser, Grèzes, Passingham, \& Haggard, 2005; Kilner, Friston, \& Frith, 2007; Pineda, Allison, \& Vankov, 2000). Moreover, it has been shown that during a joint Simon task, stimuli that referred to the partner's action evoked a similar frontal cerebral signal as the stimuli that were addressed to the participants themselves (Sebanz, Knoblich, Prinz, \& Wascher, 2006).

Some recent studies have started investigating whether action co-representation also occurs during cooperative tasks with artificial automated systems. For example, a study showed a congruency effect during a joint Simon task when participants thought they were interacting with an unseen computer (Wen \& Hsieh, 2015). However, other studies that used a similar paradigm found no congruency effect, whether at the behavioral or at the cerebral level, during a joint Simon task with an unseen computer (Tsai, Kuo, Kung, \& Tzeng, 2008) or with a robot with high level of human-like appearance (i.e. a full humanoid robot with a torso, an anthropomorphic head, eyes, and two arms with fingers) when it was described as an unintentional robot (Stenzel, Chinellato, Tirado Bou, \& del Pobil, 2012). Similarly, cerebral motor resonance has been shown not to arise during the observation of non-human-like machine-generated actions (Mann, Williams, Ward, \& Jannelle, 2007; Perani et al., 2001; but see Gazzola, Rizzolatti, Wicker, \& Keysers, 2007; Kuz, et al., 2015) while action recognition mechanisms has been shown to be sensitive to artificial humanoid avatars' actions (Ferstl, Bülthoff, de la Rosa, 2017).

In sum, these studies suggest that during joint actions people tend to spontaneously simulate the action of another human partner but not the actions of an artificial agent (excepted when people believe that the artificial partner is an intentional human-like agent). 
Moreover as we mentioned above, a separate line of evidence indicates that people can experience agency for other human's action but fail to experience it for actions performed by artificial systems. In line with these elements of evidence, the present study sought to investigate whether the ability to experience agency for the actions performed by another agent is linked to the ability to co-represent the agent's actions. To address this issue we ran a behavioral study combining a Social Simon reaction time task and an intentional binding task. The reaction times observed in the Simon task served as an index of action co-representation while IB was used as an implicit measure of the sense of agency. More specifically, participants performed the Simon task alone (standard Simon condition), or with another agent (joint go-nogo conditions) that could be another human or a computer (we used a desktop computer considering that it is the artificial system that people most commonly work with). Participants were required to estimate the time interval between their action or the action of the co-agent and a subsequent auditory outcome. This allowed us to investigate two issues. Firstly, it permitted us to assess whether participants co-represent the actions performed by a human and an automated artificial co-actor. For instance, if people do not corepresent the action performed by an automated system, we should observe the classical stimulus-response congruency effect (see description of the Simon task above) when participants perform a joint task with another human partner but not when they perform it with an artificial agent. Secondly, it allowed us to evaluate whether participants' experience of self-agency and vicarious agency during joint actions is linked to their ability to corepresent the action of their partner.

\section{Method}

\subsection{Ethic statement}

This study was approved by the CERES (recommendation $n^{\circ} 201726$, institutional ethical research committee of the Paris Descartes University, France). The investigation was carried out in accordance with the Declaration of Helsinki and each participant provided his or her written informed consent before starting the experiment. All participants were assigned a number in order to ensure the anonymity of the data.

\subsection{Participants}


Sixteen healthy adults volunteered to take part in the experiment (6 women, mean age 24.75 years, SD of age 3.15 years). One participant was excluded from the sample due to his poor time discrimination performances observed during the training session. All participants were right-handed and had normal or corrected-to-normal vision. None of them had prior knowledge about the purpose of the experiment.

\subsection{Material \& Stimuli}

Participants were sitting $46 \mathrm{~cm}$ from a computer screen with a refresh rate of $120 \mathrm{~Hz}$.

Stimulus presentation was controlled using E-prime software (2.0 version). Visual stimuli consisted of two dots of $0.5 \mathrm{~cm}$ diameter, one green and the other red. An auditory tone (1000 Hz, $200 \mathrm{~ms}$ duration), presented via a speaker placed behind the participant, was used during the experiment as the effect of the participant/partner's button press for measuring intentional binding.

\subsection{Procedure}

Participants were sitting in front of a screen and had to detect, as quickly and as accurately as possible, colored dots that appeared either to the left or to the right side of a central fixation cross. This task could be performed alone, jointly with another human (the experimenter), or jointly with a computer.

In the standard Simon condition, participants were sitting in front of the center of the screen. They were instructed to perform as quickly as possible a right key-press with their right index finger when a green dot was displayed on the screen, regardless of whether it appeared on the left or right side of the screen. When a red dot was displayed they were required to execute a left key-press with their left index finger, regardless of whether it appeared on the left or right side of the screen (Figure 1a).

In the individual go-nogo condition, participants were sitting in front of the right side of the screen and an empty chair was placed in front of the left side of the screen. They were asked to perform a right key-press with their right index finger when presented with a green dot regardless of whether it appeared on the left or right side of the screen, and to not react when presented with a red dot (Figure 1b).

In the joint conditions, participants were sitting in front of the right side of the screen. They executed a right key press with their right index finger when presented with a green dot, independently of its location on the screen. When presented with a red dot they were asked to not react. Importantly, the task could be performed jointly with another human agent (joint 
$H H$ condition, Figure 1c) or with an automated artificial system, i.e. a computer (joint $H M$ condition, Figure 1d). When the partner was a human, she was sitting on the left side of the screen and performed left key-presses with her left index finger. When the partner was the computer, an empty chair was placed in front of the left side of the screen.

In the passive observation conditions, participants were sitting in front of the right side of the screen and they had to observe another agent (another human agent in the passive observation $H$ condition and the computer in the passive observation $M$ condition) performing the red dot detection (Figure 1e \& 1d). Both the human and the automated system responded to the presentation of the red dot. When a green dot was displayed no response was required by the partner.

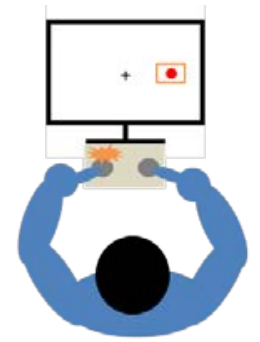

Standard Simon condition

(a)

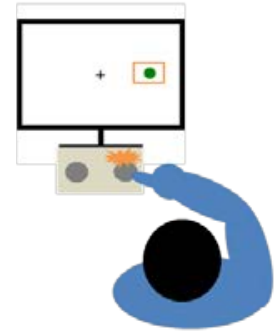

Individual go-nogo condition

(d)

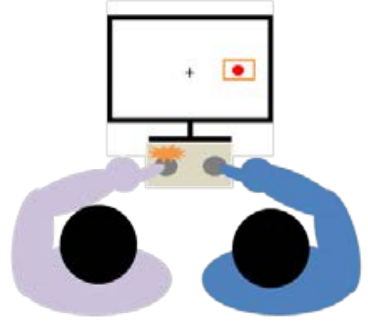

Joint $H H$ condition

(b)

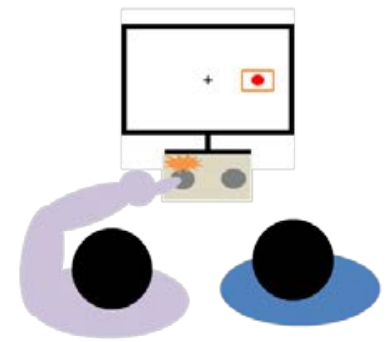

Observation $H$ condition

(e)

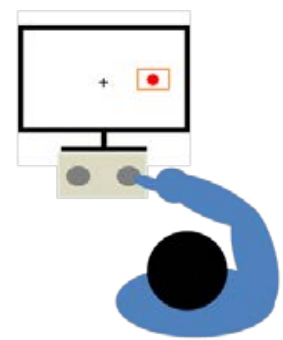

Joint HM condition

(c)

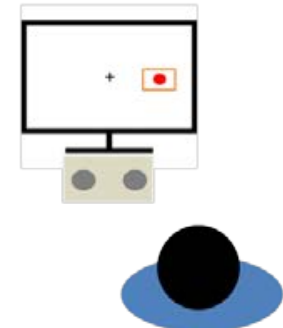

Observation $M$ condition

(f)

Figure 1. Experimental setup for the standard Simon condition (a), the human-human joint condition (b), the human-machine joint condition (c), the individual go-nogo condition (d), the human observation condition (e), and the machine observation condition (f). See text for more details.

Each trial started with a fixation cross that appeared at the center of the screen during 500 ms. Thereafter, the target immediately appeared on the screen and participants had at most 1800 ms to press their response key otherwise an error message appeared and the trial 
was canceled.

Participants were informed of the onset of their own action and the action of their coagent (human or computer) by the presentation of an empty square displayed around the target for a duration of $200 \mathrm{~ms}$. Participants were required to fixate the computer screen throughout the experiment and to not look at the actions performed by the human agent. When the human co-agent performed the target detection task, the square onset times corresponded to the coagent's real reaction times. When the computer performed the target detection task, the square onset times were taken from a normal distribution calculated from the mean and standard deviation of the human co-agent's response times computed during a pre-test session. More specifically, square onset times were selected randomly within two standard deviations of this distribution.

Correct target detections were followed by an auditory tone presented after the response at one of three possible Stimulus Onset Asynchronies (SOA) of 400, 900 or 1400 ms. The SOA for a given trial was selected randomly. After the presentation of the sound, participants had $4 \mathrm{~s}$ maximum to verbally report the perceived duration between the onset of the target detection (indicated by the square appearing around the target) and the onset of the auditory tone (see Figure 2 for a summary). This time interval estimates served as an implicit measure of participants' sense of agency. Note that participants were not asked to perform the temporal estimations in the standard Simon condition as they made estimations for selfgenerated actions in the individual go-nogo condition.

Participants were trained at the beginning of each experimental condition block to estimate and report their perceived duration of the action-tone intervals. During this training, they were presented with an empty square that flashed, followed by an auditory tone with a random delay between $200 \mathrm{~ms}$ and $2000 \mathrm{~ms}$. They had to verbally report the perceived duration of this interval in milliseconds. Then, they were given the correct delay with a visual feedback in order to accurately recalibrate their internal clock. This training session consisted in 20 trials. Thereafter, participants performed 20 trials of the given experimental condition as training. The goal was to familiarize them with the task so that they would associate their key press with the following auditory tones.

The experimental conditions were tested using a within-subject design and the order of conditions that each participant completed was selected randomly. Trials were coded as congruent when the target appeared on the side of the participant's response key, and as incongruent when the target appeared on the opposite side of the participant's response key. Participants completed a total of 3600 trials, (6 Tasks (standard Simon, individual go-nogo, 
joint $H H$, joint $H M$, passive observation $H$, and passive observation $M$ ) x 2 Targets (Green dot, Red dot) $\times 2$ Congruency levels (Congruent, Incongruent) $\times 3$ Delays $(400,900,1400) \times$ 50 trials).

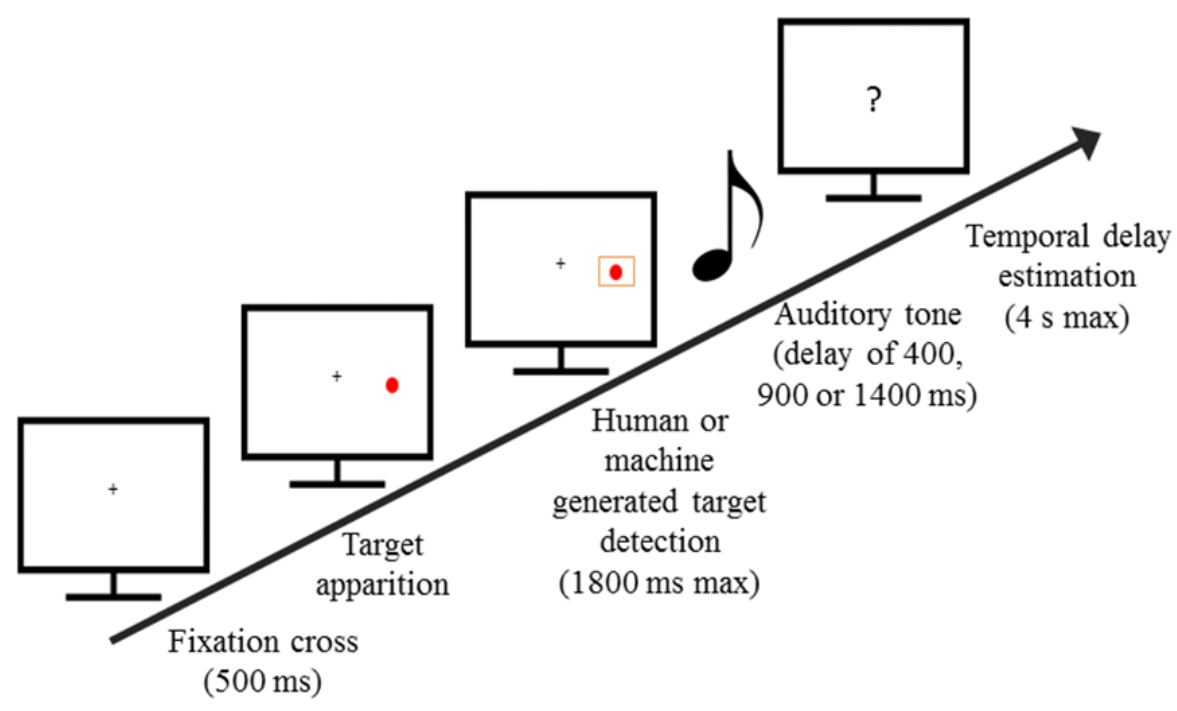

Figure 2. Trial timeline. A fixation cross appeared for $500 \mathrm{~ms}$. Then, the target appeared and the agent (the participant or the partner) had to detect it before $1800 \mathrm{~ms}$. All target detections were signaled by an empty square around the target. An auditory tone was generated at a delay of 400, 900 or $1400 \mathrm{~ms}$ after target detection. The participant had to report the temporal delay between the onset of the target detection and the onset of the tone.

\section{Data analyses}

Our dependent measures were the mean target detection Response Times (RTs) and the mean perceived action-tone temporal interval. Statistical analyses were performed with $\mathrm{R}$ software (3.3.1 version). Extreme values (the values that were below or above 2 standard deviations from the mean) of the participants' response times and perceived intervals were excluded from further analyses in order to eliminate outliers and allow for robust statistical analyses. The significance level was set at $\alpha=.05$.

To distinguish participants' trials from the partner's trials in the joint conditions, participants' trials were labeled joint HH self and joint HM self, and the partners' trials were labeled joint HH other and joint HM other.

\subsection{Response times (RTs) analysis}


This analysis was based exclusively on the data gathered in the conditions in which participants performed an action (standard Simon, individual go-nogo, joint $\mathrm{HH}$ self, and the joint $H M$ self). The analysis aimed at quantifying the Social Simon Effect (SSE) when participants interacted with a human co-agent (joint go-nogo HH self) and with the computer (joint go-nogo HM self). To investigate the effect of task order on participants' RTs we introduced the Order (1st, 2nd, 3rd, 4th, 5th, 6th) as a covariate in a linear mixed model $\left(m_{\widetilde{R T}}\right)$, with Task and Congruency as fixed factors and Subject as random effect. The factor Order indicates when a given task (e.g. standard Simon) was presented during the experiment. For instance, if the participant performed firstly the standard Simon task, we would attribute the Order " 1 st" to this task. Instead, if the standard Simon task was performed second, we would attribute the order " $2^{\text {nd," }}$ to this task and so on for the other Order values. This $\operatorname{model}\left(m_{\widetilde{R T}}\right)$ was then compared, using a likelihood ratio test, to a simpler model that included the same fixed and random effects but without order as a covariate $\left(m_{R T}\right)$ :

$$
\begin{aligned}
& m_{R T}: \mathrm{RT} \sim \text { Task } * \text { Congruency, random }=\text { Subject } \\
& m_{\widetilde{R T}}: \mathrm{RT} \sim \text { Task } * \text { Congruency }+ \text { Order, random }=\text { Subject }
\end{aligned}
$$

We found no significant difference between $m_{R T}$ and $m_{\widetilde{R T}}$ (respectively logLik = 697.94 and $\operatorname{logLik}=-695.97, \chi^{2}(5)=3.94, p=.56$ ). This indicates that including Order as a covariate did not improve our model of the observed data. Thus, Order was excluded from further analyses. We conducted a within-subjects analysis of variance (ANOVA) on reaction times with Task (standard Simon, individual go-nogo, joint HH self, joint HM self) and Congruency (Congruent, Incongruent) as factors. The Target (Green dot, Red dot) factor was not included in the ANOVA because the SSE does not rely on the target identity but rather on the congruency between the location of the target and the location of the response key. The Delay (400, 900 and 1400) factor was irrelevant for the analysis as the auditory tone was produced after participants' response and therefore could not influence their RTs.

\subsection{Temporal estimation accuracy checking}

To verify whether participants correctly perceived the different action-tone temporal intervals, we conducted a prior within-subjects one-way ANOVA on the raw temporal estimations Delay $(400,900,1400)$ as factor. This control analysis allowed us to ensure that participants paid attention to the action-sound intervals. 


\subsection{Intentional binding analysis}

This analysis aimed at investigate the influence of the co-agent on participants' agency experience. To characterize the IB phenomenon, we considered the mean perceived actiontone temporal interval in the passive observation $M$ condition as our baseline, i.e. when no agency was involved at all (see Poonian, McFadyen, Ogden, \& Cunnington, 2015; Wohlschläger, Engbert et al., 2003). We measured the IB by subtracting the mean perceived action-tone temporal interval in the passive observation $M$ condition from the mean perceived action-tone temporal interval in all other experimental conditions (negative values indicate temporal underestimations in these conditions compared to the baseline). Hence, the passive observation $M$ condition was not included as a factor in the IB analyses.

Note that for the intentional binding analyses (see below) we did not include the action - tone Delay (400, 900 and $1400 \mathrm{~ms}$ ) as a separate factor. Indeed, in the present experiment we were interested in the way the social context influences IB in general, rather than its influence on IB for different temporal intervals.

Given that the order of the experimental conditions was fully counterbalanced between participants, we introduced the Order (1st, 2nd, 3rd, 4th, 5th, 6th) factor as a covariate in a linear mixed model $\left(m_{\widetilde{I B}}\right)$ with Action Context (Human individual action, Human-human joint action, Human-computer joint action), Agent (Self, Other) and Congruency (Congruent, Incongruent) as fixed factors and Subject as random effect. The Action Context referred to the social context in which the Simon task was performed, i.e. whether the agent was acting alone (Human individual action), co-acting with another human (Human-human joint action), or with a computer (Human-computer joint action). Accordingly, the Human individual action context included the passive observation $H$ for the actions that were performed by the human partner and the individual go-nogo condition for the actions performed by the participant him/herself. Note that there was no temporal estimation measurement in the standard Simon condition. That is why this condition was not included in the analyses of the IB phenomenon. The Agent (Self, Other) factor referred to the author of the action, i.e. the participants themselves or their partner respectively (see Table 1). Using a likelihood ratio test, we then compared this model $\left(m_{\widetilde{I B}}\right)$ with another mixed model $\left(m_{I B}\right)$ with only Action context, Agent, and Congruency as fixed factors and Subject as random effect:

$$
\begin{aligned}
& m_{I B}: \text { IB } \sim \text { Action Context } * \text { Agent } * \text { Congruency, random }=\text { Subject } \\
& m_{\overparen{I B}}: \text { IB } \sim \text { Action Context } * \text { Agent } * \text { Congruency }+ \text { Order, random }=\text { Subject }
\end{aligned}
$$


The analysis showed that the $m_{\widetilde{I B}}$ model including Order as a covariate fitted our data better than the $m_{I B}$ model (respectively logLik $=-1135.63$ and $\log \operatorname{Lik}=-1154.96, \chi^{2}(5)=$ 38.66, $p$ <.001). Hence, we conducted a within-subjects ANOVA on IB with Action Context (Human individual action, Human-human joint action, Human-computer joint action), Agent (Self, Other) and Congruency (Congruent, Incongruent) as fixed factors and Order (1st, 2nd, 3rd, 4th, 5th, 6th) as a covariate.

Factor: Action Context

Human individual Human-human joint Human-computer

\begin{tabular}{lllll} 
& \multicolumn{2}{c}{ action } & action & joint action \\
\hline \multirow{3}{*}{ Factor : Agent } & Self & Individual go-nogo & Joint HH self & Joint HM self \\
\cline { 2 - 4 } & Other & $\begin{array}{l}\text { Passive } \\
\text { observation } H\end{array}$ & Joint HH other & Joint HM other \\
& & & \\
\hline
\end{tabular}

Table 1. Factorial design for the intentional binding analysis

\section{Results}

\subsection{Social Simon Effect}

We first examined the SSE during joint-actions with another human and with a desktop computer (Figure 3). We assessed the normality of the RTs distributions of the differences between the congruent trials and the incongruent trials in the standard Simon, individual go-nogo, joint $\mathrm{HH}$ self and joint HM self conditions using the Shapiro-Wilk test. The analyses showed that none of the RTs distribution deviated from normality (all $W>.90$ and all $p>.10$ ). We then computed a within-subjects $4 \times 2$ analysis of variance (ANOVA) with the factors Task (standard Simon, individual go-nogo, joint HH self, joint HM self) and Congruency (Congruent, Incongruent). We found a significant main effect of Congruency indicating longer mean RTs on Incongruent trials compared to Congruent trials $(F(1,14)=$ 5.47, $p=.03$ ) but no significant main effect of Task $(F(3,42)=.80, p=.50$, ns $)$. We found a significant interaction between Congruency and Task $(F(3,42)=9.99, p<.001)$. Post-hoc comparisons using Tukey's HSD test revealed that mean RTs on Incongruent trials was significantly longer than the mean RTs on Congruent trials in the standard Simon condition 
(respectively $426.19 \mathrm{~ms}$ and $409.25 \mathrm{~ms}, p=.001$ ) and in the joint $\mathrm{HH}$ self condition (respectively $390.23 \mathrm{~ms}$ and $403.51 \mathrm{~ms}, \mathrm{p}=.02$ ) but not in the individual go-nogo condition (respectively $414.32 \mathrm{~ms}$ and $416.09 \mathrm{~ms}, p=.99$, ns) nor in the joint HM self condition (respectively $434.78 \mathrm{~ms}$ and $444.16 \mathrm{~ms}, \mathrm{p}=.23$, ns). Hence, we observed the classical Simon Effect in the standard Simon and when participants performed the task with another human agent (joint $\mathrm{HH}$ self). No Simon effect was observed when participants interacted with a machine.

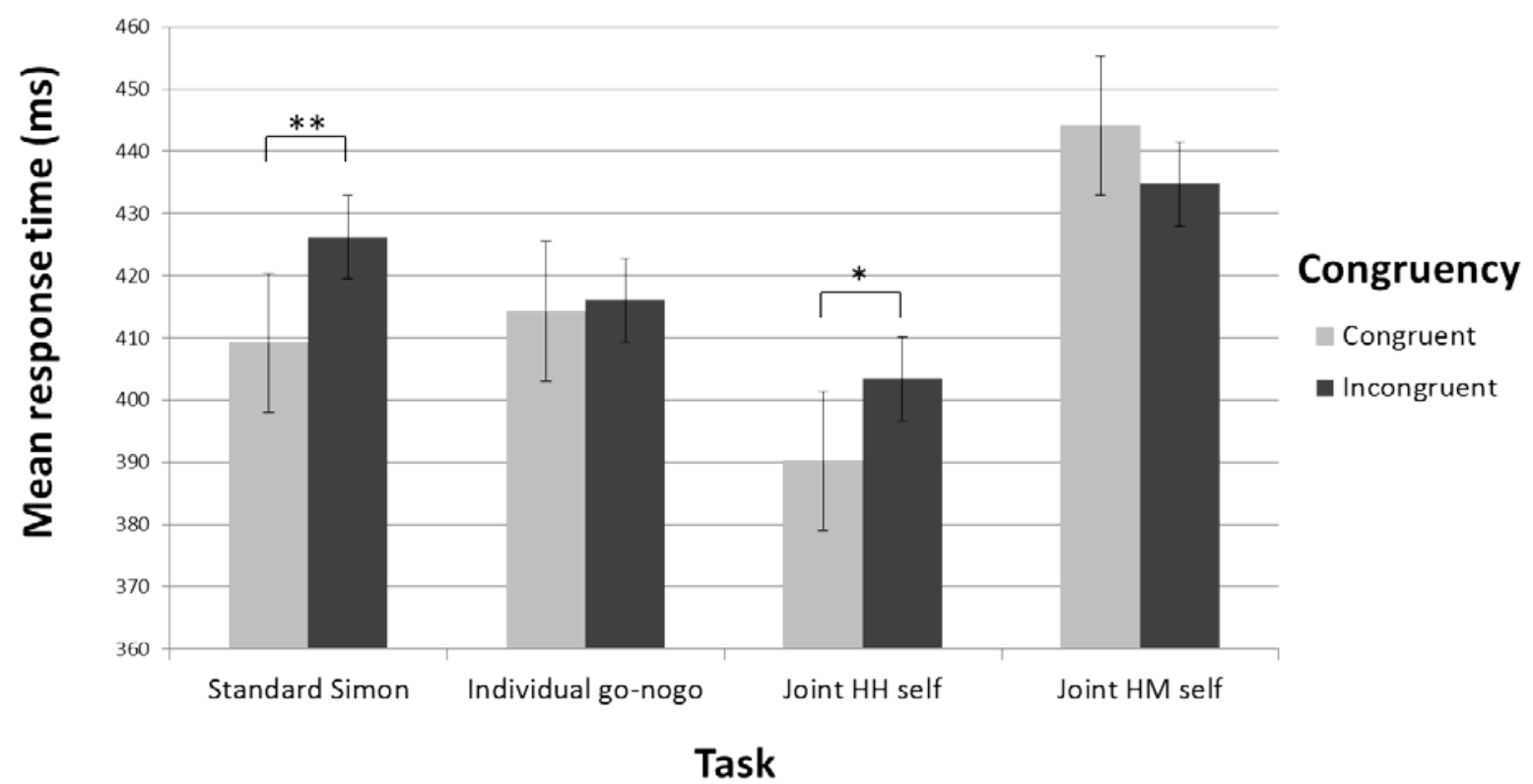

Figure 3. Congruency effect for the standard Simon, individual go-nogo, and joint conditions. Error bars represent standard errors.

\subsection{Temporal estimation accuracy checking}

We verified whether participants correctly perceived the different action-tone temporal intervals that were used in the task by assessing whether they adjusted their temporal estimations accordingly. We investigated the effect of Delay on the mean perceived actiontone temporal interval. We computed a within-subjects one-way ANOVA with the factor Delay $(400,900,1400)$. We found a significant main effect of Delay on the perceived actiontone temporal interval estimations $(F(2,28)=316.71, p<.0001)$. Post-hoc comparisons using Tukey's HSD test revealed that individuals reported significantly higher temporal intervals when the Delay between the action and the tone was 1400 ms compared to when the delay was $900 \mathrm{~ms}$ (respectively $1133.04 \mathrm{~ms}$ and $797.83 \mathrm{~ms}, p<.0001$ ) and when the delay was 400 ms (433.57 ms, $p<.0001)$. In addition, individuals reported higher temporal intervals with a 
900 ms delay compared to a 400 ms delay $(p<.0001)$. Even though this was not a variable of interest (indeed, we only introduced various delays in order to avoid the predictability bias), this effect demonstrated that participants were paying attention to the temporal delays and correctly discriminated the different delays.

\subsection{Intentional Binding}

Firstly, we calculated the amount of IB by subtracting the mean perceived action-tone temporal interval in the passive observation $M$ condition from the mean perceived action-tone temporal interval in all the other conditions. We computed a within-subjects $3 \times 2 \times 2$ ANOVA Action Context (Human individual action, Human-human joint action, Humancomputer joint action), Agent (Self, Other) and Congruency (Congruent, Incongruent) as fixed factors and Order (1st, 2nd, 3rd, 4th, 5th, 6th) as a covariate.

We found neither a main effect of Agent $(F(1,14)=.11 p=.74, n s)$ nor a main effect of Congruency $(F(1,14)=0.38, p=.54, n s)$. However, we found a significant main effect of Action Context $(F(2,28)=10.78, p<.001)$. Post-hoc comparisons using Tukey's HSD test investigated this main effect and revealed that participants exhibited greater IB for human individual actions context than for human-machine joint actions context $(p<.001)$. In addition, there was no difference on IB between the human individual actions context and the human-human joint actions context $(p=.24, n s)$. Finally, participants exhibited greater IB for human-human actions context than for human-machine joint actions context $(p<.001)$.

Furthermore, we found a significant Action Context $\mathrm{x}$ Agent interaction $(F(2,28)=$ 11.24, $p<.001$ ) on IB (Figure 4). Considering that Congruency did not interact with the other factors, we averaged congruent and incongruent trials for further analyses.

Post-hoc comparisons using Tukey's HSD test revealed that IB for self-generated actions was greater when participants were acting alone (individual go-nogo condition) compared to when they were jointly acting with a machine $(p<.001)$. However, IB for selfgenerated actions did not differ when participants were performing the task alone (individual go-nogo condition) compared to when they were interacting jointly with another human agent $(p=.34)$. Similarly, IB did not differ when participants acted with another human agent compared to when they interacted with a machine, although there was a trend $(p=.06, n s)$. Accordingly, this indicates that IB observed for self-generated actions did not differ whether participants performed the task alone or interacted with another human agent but tended to strongly decrease when participants interacted with a machine. 
Additional post-hoc comparisons using Tukey's HSD test on IB for other-generated actions revealed greater IB when participants performed the task with another human agent compared to when they were observing the same agent completing the task alone (passive observation $H$ condition) $(p<.001)$. In addition, we observed greater IB for other-generated actions when participants performed the task jointly with a human agent compared to when they performed it with the computer $(p<.001)$. However, there was no difference in IB for other-generated actions when participants observed a human agent performing the task alone (passive observation $H$ condition) compared to when they interacted with the computer ( $p=$ $.81, n s)$. Accordingly, this indicates that the IB observed for other-generated actions strongly increased only when participants performed the task together with a human agent compared to the mere passive observation of the same action. This enhancement was not observed during joint actions with a machine.

Further post-hoc comparisons using Tukey's HSD test showed greater IB for selfgenerated actions compared to other-generated actions when participants and the other human agent were performing the task alone (human individual action context) $(p=.005)$. During human-human joint actions, IB was weaker for self-generated actions compared to othergenerated action $(p=.03)$. There was no difference on IB between self- and other-generated actions when participants interacted with a machine $(p=1.00, n s)$. In addition, no difference was observed between the IB for self-generated actions performed alone and the IB for othergenerated actions in the context of human-human joint action ( $p=.95, n s)$. In sum, participants reported stronger IB when they performed the task alone compared to when they passively observed another person performing the same action. However, during humanhuman joint action, this trend was reversed. Notably, they reported stronger IB for the action of their partner than for their own action. 


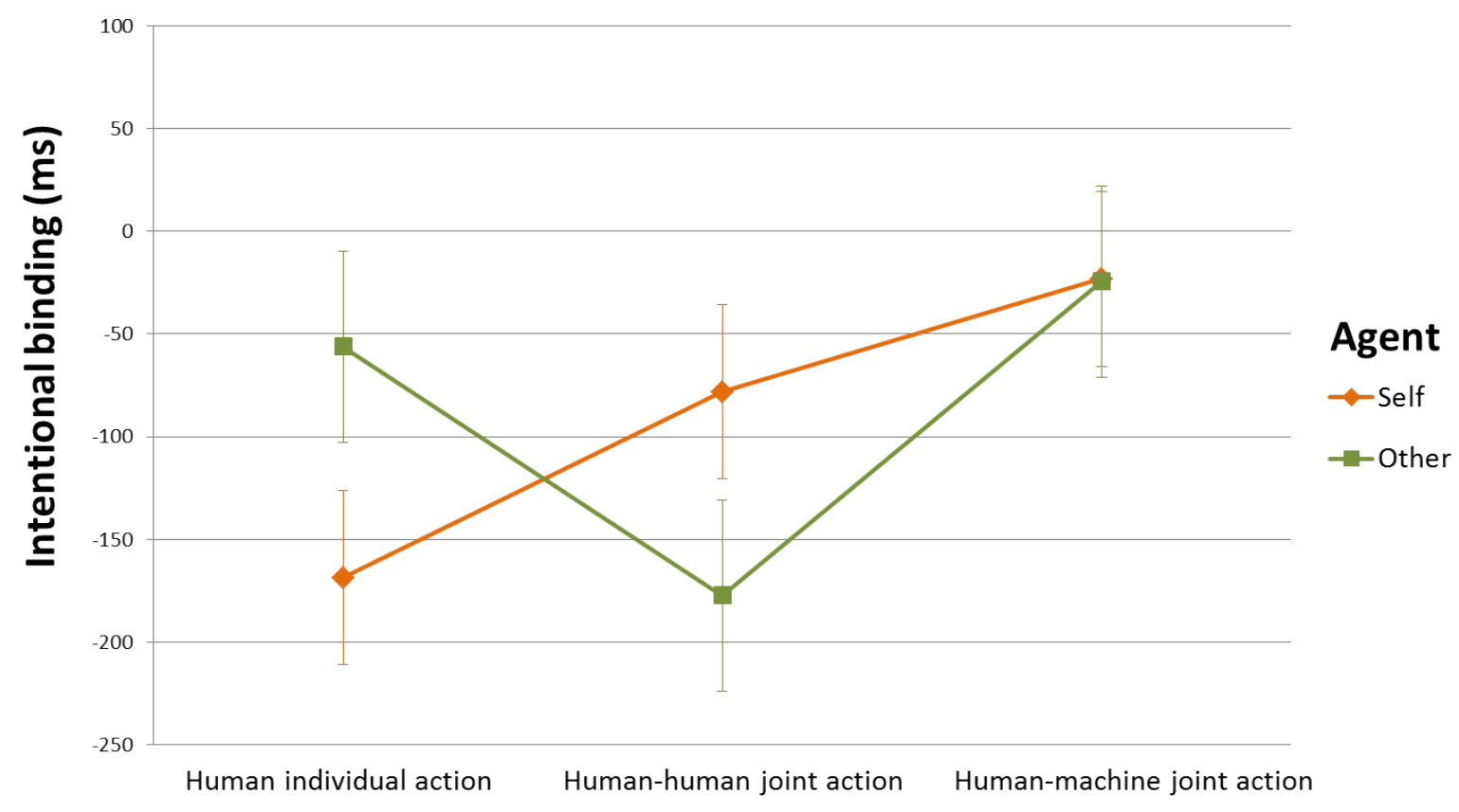

Action context

Figure 4. Intentional binding for self- and other-generated actions in the action contexts of human individual action, human-human joint action, and human-machine joint action. The vertical bars represent the standard error values.

\section{Discussion}

In this study, our aim was to investigate the sense of agency in joint action with another human versus a desktop computer. To assess action co-representation, we used a typical Social Simon task where participants had to detect a target that could appear on the same side as their response key or on the opposite side. Accurate target detection triggered an auditory tone after a randomized delay. Participants had to estimate the temporal delay between the target detection and the onset of the tone. This estimate served as an implicit measure of participants’ sense of agency (Haggard, et al., 2002).

We found the classical Simon effect with longer response times in incongruent trials when participants performed a double target detection task (standard Simon condition) but not when they performed a single target detection task (individual go-nogo (self) condition). Moreover, we found a Social Simon Effect (SSE) when the participants performed a single target detection task jointly with another human agent performing the complementary action (joint go-nogo HH (self) condition). This suggests that, during human-human interactions, 
participants experienced the action performed by a human partner as if it was their own action. However, this effect disappeared when a computer program replaced the human partner. These findings are consistent with previous results supporting the claim that the social identity of the partner critically influences individuals' action co-representation abilities during a joint task (Stenzel, et al., 2012; Wen \& Hsieh, 2015). One possible explanation is that the artificial agent was not considered as an agent either because it was not perceived as acting intentionally or because the causal relationship between the agent and the action effect was opaque or both. Indeed, a previous study investigating the SSE during interactions with a humanoid robot failed to observe this effect when the robot was described as an unintentional device that passively executed a deterministic command whereas the SSE was present when participants were induced to believe that the robot was an intentional active and intelligent human-like agent (Stenzel,et al.,2012). In addition, it has been proposed that the perceived coagent's agency plays an important role in the emergence of SSE (Stenzel, Dolk, Colzato, Sellaro, Hommel, \& Liepelt, 2014). In their study, Stenzel and colleagues (2014) asked participants to perform a joint Simon task next to a co-agent who either intentionally performed the response key presses (agency+/intentionality+ condition), or passively placed their finger on the response key that automatically moved up and down (agency/intentionality- condition), or who was thought to controlled the response key with a braincomputer interface while placing their finger besides the response key (agency/intentionality+ condition). The authors found that the SSE was manifested only in the agency+/intentionality+ condition, that is to say, when the causality between the co-agent and the action effect was not disrupted. Hence, perceived agency may play an important role in the SSE (Stenzel, et al. 2014).

Note that the "social" component of the SSE has been recently criticized (Dolk, et al., 2011; Dolk, et al., 2013, for a review see Dolk, et al., 2014). Instead, a referential-coding approach has been proposed according to which salient events, such as a physical presence for example - whatever social or not -, serve as a spatial referential for action coding. When considering the referential-coding approach, one can argue that the human partner in our experiment may have been more salient than the computer partner and could thus have grabbed the attention of the participant to a higher degree, influencing participants' response times. Our study was not designed to and cannot provide an answer to this debate. However, we believe that the referential-coding approach cannot explain the influence of social factors on the SSE. For example, it has been shown that individuals showed less or even no SSE when they were in a competitive context compared to a cooperative context (Iani, Anelli, 
Nicoletti, Arcuri, \& Rubichi, 2011; Ruissen \& de Bruijn, 2016) or after a negative affect induction compared to a positive affect induction between the two co-agents (Hommel, Colzato, van den Wildenberg, 2009; Kuhbandner, Pekrun, \& Maier, 2010) although the participants performed the very same task. This theoretical account also hardly explains the difference found between intentional and non-intentional artificial agents (Stenzel et al., 2012). We believe that this issue could be settled by further studies combining brain imaging and behavioral techniques.

Interestingly, we observed that IB phenomenon for self-generated actions remained stable regardless of whether participants performed the task alone or with another human agent. However, IB decreased strongly when participants interacted with a machine. This pattern of results indicates that the social context played a pivotal role in implicit self-agency attribution as measured by the intentional binding phenomenon. Even though participants in the present experiment had to perform the same action leading to the same sensory effect in all conditions, they tended to bind their own action and its effect differently according to the social context. The intentional binding for their action was maximal when they were performing the task alone. It decreased drastically as soon as they were collaborating with a machine. Thus, sharing a task with an artificial agent seems to decrease the sense of selfagency. This result corroborate previous findings showing a reduction of self-agency during a human-human cooperative task compared to an individual task, even when there was no ambiguity about who had caused the outcome (Beyer, Sidarus, Bonicalzi, \& Haggard, 2017). Our study provides however original evidence that this process of diffusion of responsibility does mainly occur during human-computer interaction, suggesting that when interacting with a machine our sense of responsibility strongly decreases.

More importantly, the vicarious sense of agency for the partner's action disappeared when the partner was the computer, as did the congruency effect on response times. Hence, the IB phenomenon seemed to parallel the SSE effect when comparing the human-human condition with the human-machine condition. This lends support to the view that vicarious sense of agency and SSE could potentially share common underlying mechanisms, but this needs to be confirmed by neuroimaging studies. As mentioned earlier, there are currently two main hypotheses explaining the SSE. The first more classical account posits that we automatically activate our own sensorimotor representations when partnered with a human coagent (Sebanz, et al., 2003). The second theory accounting for the SSE suggests that action coding and thus decision is influenced by salient environmental cues (Dolk, et al., 2011; Dolk, 
et al., 2013). However, a third explanation combining the two accounts could also be taken into consideration, explaining both the RTs and the IB results. In the case of individual selfgenerated actions, individual's attention was focused on the ongoing task and their sensorimotor network was activated, leading to a greater sense of agency. During the joint tasks, the need of participants to coordinate their actions with their partner induced an attentional shift toward the other-generated actions, which in turn engaged the participant's sensorimotor network when the partner was a human being, leading to a vicarious sense of agency in the specific context of the human-human joint task. Finally, during the humanmachine joint task, the absence of an intentional partner brought the participants to pay less attention to the actions performed by the machine. As a consequence, participants' sensorimotor network was not activated in this case, leading also to a decrease of agency for the actions generated by the machine. However, further studies are needed to investigate which hypothesis provides the best account for the outcomes reported here. Taken together these results suggest that participants experienced vicarious agency during joint actions with other humans but not with machines. As for now, the findings might be considered to provide further evidence in favor of the view that the human representational system is biologically tuned (Tsai \& Brass, 2007).

Other interesting results were observed in our study. First, IB for the actions generated by a human partner was stronger during joint actions compared to mere passive observation of the same action. These results might suggest that being involved in a joint task leads to a stronger representation of the agency of the co-actor. Second, we found that during the human-human collaborative condition, IB for the co-agent's action was stronger compared to IB for the participant's own actions. This outcome contrasts with the experiment reported by Obhi \& Hall (2011a), in which intentional binding was the same for the participant's and the partner's action when they were performing a joint task. This difference might depend on the fact that Obhi \& Hall (2011a) used a very different design compared to our study. In their experiment, the two participants were required to press the same button jointly, whereas in our experiment the participant and their partner pressed different buttons at different times in response to different stimuli. The way agency is allocated among partners may depend on the type of collaboration required by the task. When a participant and a co-actor are required to perform an action together (as in Obhi \& Hall, 2011a), the IB observed might reflect a summation of both self- and other- agency. However, in our case since the participant's and the partner's action were separate in time, we believe that the IB we observed for the actions generated by the co-actor reflect a sense of vicarious agency. Finally, we did not observe any 
effect of Congruency on participants' sense of agency. This is in contrast with past studies showing that the fluency of action selection modulates the sense of agency (Chambon \& Haggard, 2012; Sidarus \& Haggard, 2016). Notably, past research showed that when action selection is facilitated by the presentation of a cue, individuals report higher judgement of control compared to when action selection is not facilitated. In our study we did not find any effect of action facilitation on participants' sense of agency. However, unlike previous experiment on the fluency of action, we did not use an explicit rating scale but an implicit measure of agency. This might suggest that intentional binding and explicit judgments of agency do not share the same processes (Dewey \& Knoblich, 2014).

\section{Conclusion}

We raised the question of the relationship between action co-representation and the sense of we-agency. Given the convergence of the SSE and the vicarious sense of agency, we hypothesized that as soon as individuals are able to use their own motor system to simulate their partner's action into their own motor system, they can understand their partner's intention with the help of their previous experiences, supporting the construction of a vicarious sense of agency. During human-machine interactions, as individuals do not consider the artificial system as intentional, they do not use their sensori-motor system to simulate and try to understand the machine-generated action and consequently are unable to develop a vicarious sense of agency. This leads to the question of how we can design artificial automated systems that we can understand and thus guarantee a meaningful human-machine interaction. To address this issue an increasing number of studies are now investigating whether action co-representation is facilitated by humanized automated artificial systems (for a review, see Sahaï, Pacherie, Grynszpan, \& Berberian, 2017). Human-like automata could more easily be considered as intentional agents and may facilitate action co-representation and in turn ensure optimal human-machine interactions. In line with this notion, it has been shown that humanized machines could enhance the individuals' sense of agency compared to traditional automata (Wohlschläger, Haggard, et al., 2003).

\section{Author contribution}


All authors listed have made a substantial, direct and intellectual contribution to the work, and approved it for publication.

\section{Funding source}

This work was funded by a French National Research Agency grant (Young researcher program - ANR-15-CE26-0010-01). Elisabeth Pacherie’s work was supported by ANR-10LABX-0087 IEC and ANR-10-IDEX-0001-02 PSL*.

\section{Conflict of Interest Statement}

The authors declare that the research was conducted in the absence of any commercial or financial relationships that could be construed as a potential conflict of interest.

\section{References}

Berberian, B., Sarrazin, J-C., Le Blaye, P., and Haggard, P. (2012). Automation technology and sense of control: a window on human agency. PLoS One 7(3), e34075, https://doi.org/10.1371/journal.pone.0034075

Beyer, F., Sidarus, N., Bonicalzi, S., and Haggard, P. (2017). Beyond self-serving bias: diffusion of responsibility reduces sense of agency and outcome monitoring. Social Cognitive and Affective Neuroscience 12(1), 138-145. doi:10.1093/scan/nsw160

Calvo-Merino, B., Glaser, D., E., Grèzes, J., Passingham, R., E., and Haggard P. (2004). Action observation and acquired motor skills: an fMRI study with expert dancers. Cerebral Cortex 15, 1243-1249. doi: 10.1093/cercor/bhi007

Capozzi, F., Becchio, C., Garbarini, F. Savazzi, S., and Pia, L. (2016). Temporal perception in joint action: this is MY action. Consciousness and Cognition 40, 26-33.

Caspar, E., A., Christensen, J., F., Cleeremans, A., and Haggard P. (2016). Coercion changes the sense of agency in the human brain. Current Biology 26, 585-592. doi:

10.1016/j.cub.2015.12.067

Chambon, V., and Haggard, P. (2012). Sense of control depends on fluency of action selection, not motor performance. Cognition 125(3), 441-451.

https://doi.org/10.1016/j.cognition.2012.07.011 
Dewey, J., A., and Knoblich, G. (2014). Do implicit and explicit measures of the sense of agency measure the same thing? PLoS One 9(10), e110118. doi:

10.1371/journal.pone.0110118

Dolk, T., Hommel, B., Colzato, L., S., Schütz-Bosbach, S., Prinz, W., and Liepelt, R. (2011) How "social" is the social Simon effect?. Frontiers in Psychology 2(84). doi: 10.3389/fpsyg.2011.00084

Dolk, T., Hommel, B., Prinz ,W., and Liepelt, R. (2013). The (not so) social simon effect: A referential coding account. Journal of Experimental Psychology: Human Perception and Performance 39, 1248-1260. doi: 10.1037/a0031031

Dolk, T., Hommel, B., Colzato, L., S., Schütz-Bosbach, S., Prinz, W., \& Liepelt, R. (2014). The Joint Simon Effekt: A review and theoretical integration. Frontiers in Psychology 5

Ferstl, Y., Bülthoff, H., and de la Rosa, S. (2017). Action recognition is sensitive to the identity of the actor. Cognition, 166, 201-206. doi: 10.1016/j.cognition.2017.05.036

Frith, C., D. (2014). Action, agency and responsibility. Neuropsychologia 55, 137-142. doi: 10.1016/j.neuropsychologia.2013.09.007

Gazzola, V., Rizzolatti, G., Wicker, B., and Keysers C. (2007). The anthropomorphic brain: the mirror neuron system responds to human and robotic actions. Neuroimage 35, 1674-1684. doi: 10.1016/j.neuroimage.2007.02.003

Haggard, P. (2017). Sense of agency in the human brain. Nature Reviews Neuroscience 18, 196-207. doi: 10.1038/nrn.2017.14

Haggard, P., and Chambon, V. (2012). Sense of agency. Current Biology 22(10), R390-R392. https://doi.org/10.1016/j.cub.2012.02.040

Haggard P., Clark S., and Kalogeras J. (2002). Voluntary action and conscious awareness. Nature Neuroscience 5, 382-385. doi: 10.1038/nn827

Hommel, B., Colzato, L., S., and van den Wildenberg, W., P., M. (2009). How Social Are Task Representations? Association for Psychological Science 20(7).

Iani, C., Anelli, F., Nicoletti, R. Arcuri, L., and Rubichi, S. (2011). The role of group membership on the modulation of joint action. Experimental Brain Research 211, 439-445. doi: 10.1007/s00221-011-2651-x

Khalighinejad, N., Bahrami, B., Caspar, E., A., and Haggard, P. (2016). Social transmission of experience of agency: An experimental study. Frontiers in Psychology 7. doi: 10.3389/fpsyg.2016.01315

Kilner, J., M., Friston K., J., and Frith, C., D. (2007). Predictive coding: an account of the mirror neuron system. Cognitive Processing 8, 159-166. doi: 10.1007/s10339-007-0170-2 
Kuhbandner, C., Pekrun, R., and Maier, M., A. (2010). The role of positive and negative affect in the "mirroring” of other persons' actions. Cognition and Emotion 24(7), 1182-1190. doi: 10.1080/02699930903119196

Kunde, W., Weller, L., and Pfister, R. (2017). Sociomotor action control. Psychonomic bulletin \& review. doi: 10.3758/s13423-017-1316-6

Kuz, S., Petruck, H., Heisterüber, M., Patel, H., Schumann, B., Schlick, C., M., et al. (2015). Mirror neuronsand human-robot interaction in assembly cells. Procedia Manufacturing 3, 402-408. doi: 10.1016/j.promfg.2015.07.187

Mann, D., T., Williams, A., M., Ward, P., and Janelle, C., M. (2007). Perceptual-cognitive expertise in sport: a meta-analysis. Journal of Sport and Exercise Psychology 29, 457-478. doi: 10.1123/jsep.29.4.457

Moore, J., W., and Obhi, S., S. (2012). Intentional binding and the sense of agency: a review. Consciousness and Cognition 21(1), 546-561. doi: 10.1016/j.concog.2011.12.002

Moretto, G., Walsh, E., and Haggard, P. (2011). Experience of agency and sense of responsibility. Consciousness and Cognition 20, 1847-1854. doi:

10.1016/j.concog.2011.08.014

Obhi, S., S., and Hall, P. (2011a). Sense of agency and intentional binding in joint action. Experimental Brain Research 211(3-4), 655-662. doi: 10.1007/s00221-011-2675-2

Obhi, S., S., and Hall P. (2011b). Sense of agency in joint action: influence of human and computer co-actors. Experimental Brain Research 211(3-4), 663-670. doi:10.1007/s00221011-2662-7

Perani, D., Fazio, F., Borghese, N., A., Tettamanti, M., Ferrari, S., Decety, J., et al. . (2001). Different brain correlates for watching real and virtual hand actions. Neuroimage 14, 749-758. doi : 10.1006/nimg.2001.0872

Pfister, R., Obhi, S., Rieger, M. \& Wenke, D. (2014). Action and perception in social contexts: Intentional binding for social action effects. Frontiers in Human Neuroscience 8, doi: 10.3389/fnhum.2014.00667

Pineda, J., A., Allison, B., Z., and Vankov, A. (2000). The effects of self-movement, observation, and imagination on mu rhythms and readiness potentials (RP's): toward a braincomputer interface (BCI). IEEE Transactions on Rehabilitation Engineering 8(2), 219-222. doi: 10.1109/86.847822

Poonian, S., K., McFadyen, J., Ogden, J., Cunnington, R. (2015). Implicit agency in observed actions: evidence for N1 suppression of tones caused by self-made and observed actions. Journal of Cognitive Neuroscience 27, 752-764. doi:10.1162/jocn_a_00745

Ruissen, M. I., \& de Bruijn, E. R. (2016). Competitive Game Play Attenuates Self-Other Integration during Joint Task Performance. Frontiers in Psychology, 7(274). 
Sahaï, A., Pacherie, E., Grynszpan, O., and Berberian, B. (2017). Predictive mechanisms are not involved the same way during human-human vs. human-machine interactions: a review. Frontiers in Neurorobotics 11(52). doi: 10.3389/fnbot.2017.00052

Sebanz, N., Knoblich, G., and Prinz W. (2003). Representing others' actions: just like one's own? Cognition 88, B11-B21. doi: 10.1016/S0010-0277(03)00043-X

Sebanz, N., Knoblich, G., Prinz W., and Wascher, E. (2006). Twin peaks: and ERP study of action planning and control in coacting individuals. Journal of Cognitive Neuroscience 18(5), 859-870. https://doi.org/10.1162/jocn.2006.18.5.859

Sidarus, N., and Haggard, P. (2016). Difficult action decisions reduce the sense of agency: a study using the eriksen flanker task. Acta Psychologica 166, 1-11. doi:

10.1016/j.actpsy.2016.03.003

Simon, J., R., and Wolf, J., D. (1963). Choice reaction time as a function of angular stimulusresponse correspondence and age. Ergonomics 6(1), 99-105.

http://dx.doi.org/10.1080/00140136308930679

Stenzel, A., Chinellato, E., Tirado Bou, M., A., del Pobil, Á., P., Lappe, M., and Liepelt, R. (2012). When humanoid robots become human-like interaction partners: corepresentation of robotic actions. Journal of Experimental Psychology: Human Perception and Performance 38, 1073-1077. doi: 10.1037/a0029493

Stenzel, A., Dolk, T., Colzato, L., S., Sellaro, R., Hommel, B., and Liepelt, R. (2014). The joint Simon effect depends on perceived agency, but not intentionality, of the alternative action. Frontiers in Human Neuroscience 8. doi: 10.3389/fnhum.2014.00595

Strother, L., House, K., A., and Obhi, S., S. (2010). Subjective agency and awareness of shared actions. Consciousness and Cognition 19, 12-20. doi:10.1016/j.concog.2009.12.007

Tsai, C-C., Kuo, W-J., Hung, D., L., and Tzeng, O., J., L. (2008). Action co-representation is tuned to other humans. Journal of Cognitive Neuroscience 20(11), 2015-2024. doi:

10.1162/jocn.2008.20144

Wen, T., and Hsieh, S. (2015). Neuroimaging of the joint Simon effect with believed biological and non-biological co-actors. Forntiers in Human Neuroscience 9(483), doi: 10.3389/fnhum.2015.00483

Wohlschläger, A., Engbert, K., and Haggard P. (2003). Intentionality as a constituting condition for the own self-and other selves. Consciousness and Cognition 12, 708-716. doi:10.1016/S1053-8100(03)00083-7

Wohlschläger, A., Haggard, P., Gesierich, B., and Prinz, W. (2003). The perceived onset time of self-and other-generated actions. Psychological Science 14(6), 586-591. doi:

10.1046/j.0956-7976.2003.psci_1469.x 
Yamaguchi, M., Wall, H., J., and Hommel, B. (2018). Sharing tasks or sharing actions? Evidence from the joint Simon task. Psychological Research 82, 385-394. doi:

10.1007/s00426-016-0821-y 